\title{
THE INVERSE PROBLEM FOR COMPARTMENTAL SYSTEMS
}

\author{
John A. JACQUEZ \\ Departments of Biostatistics and Physiology, The University of Michigan, Ann Arbor, MI 48109, U.S.A.
}

\begin{abstract}
The current state of the inverse problem for compartmental systems is reviewed and analyzed in terms of three major phases, viz. model specification, identifiability and parameter estimation. Emphasis is given to a review of identifiability for linear compartmental systems and the need to put identifiability in perspective in the context of the inverse problem.
\end{abstract}

\section{INTRODUCTION}

In its general sense the inverse problem is the scientific process that we use in trying to unravel the nature of the world around us. Its main stages are hypothesis formulation, design of experiments to test our hypotheses, experimentation and analys is of experimental results. In light of the new knowledge gained the process is then repeated. I shall restrict my discussion to biological systems and specifically to compartmental systems but much of the material which follows is extendable to other systems. Furthermore, I shall refer primarily to 1 inear time-invariant systems but when possible will add remarks about nonlinear syst ems.

The nature of the inverse problem for linear compartmental systems stands out most clearly when contrasted with the forward problem. In the forward problem we are given the structure of the compartmental system, i.e. the number of compartments with their connections including inputs and outputs, and the values of the fractional transfer coefficients and the input functions. The forward problem starts with the system structure known and all parameter values specified and requires us to calculate the behavior of the system. In the inverse problem some of the parameters are unknown or some parameters and structure are unknown and we want to work backwards. I like to break the inverse problem into the following stages, model specification, definition of the allowed experiments, identifiability, parameter estimation, experimentation and analysis and model checking. Again, this is an iterative process. There is some variation in the breakdown into stages given by different authors but I think there is good agreement on the content and the major stages. For this paper I focus on model specification, identifiability and parameter estimation.

One of the stages mentioned above may be unfamiliar to scientists from the physical sciences, the definition of the allowed experiments. For biological systems it is invariably true that only a few of the conceptually possible experiments can actually be done. This is so because not all parts (compartments) of the system are available for signal input or for observation without severely damaging the system, a sort of biological uncertainty principle. In addition ethical and humane considerations limit the allowed experiments for some biological systems.

\section{MODEL SPECIFICATION}

The first problem is to decide whether a compartmental model is appropriate or useful. We now have available such an extensive body of theory about compartmental systems that it is often tempting to try to fit a problem into the compartmental mold. The decision should be based on detailed knowledge of the biology, of the anatomy, biochemistry and physiology of the system and problem. Like all models compartmental models are approximations but one can distinguish two extremes which differ in the nature of the approximations. The first might, for emphasis, be called compartmental models of compartmental systems. The system has an obvious partitioning into a compartmental type of structure, spacewise or in terms of chemical species or both, and the model is an approximation because mixing within the presumed compartments is not instantaneous, nor are the transfers between the compartments instantaneous. The compartmental model is a good approximation if mixing within the presumptive compartments is rapid in relation to the transfers between them and it is poor if it is the other way around. The other extreme is one in which a compartmental model is used as a discrete approximation to a continuous system. Now there is no natural partitioning of the system to guide compartmentation; compartmentation is just one way of discretizing. Such models usually have a repetitive structure determined by the nature of the system and the approximation but the size, the number of repetitive units, increases as the approximation is improved. The important point is that the size is not uniquely specifiable except as a measure of the 
closeness of approximation. I assume that these considerations have been addressed and that there is reason to choose a compartmental model. For compartmental systems model specification means the determination of the connectivity diagram of the system, i.e. the structure, the number of compartments, their connections and the inputs and out puts. The main point to make here is that this phase of the inverse process depends strongly on our knowledge of the structure and function of the biological system. The non-biologist tends to overemphasize the importance of looking for the number of exponential modes of decay in determining the structure of the system. In general the number of exponential decays is less than or equal to the order of the system. But when we take into account the experimental error a choice of sample times is in fact a choice of eigenvalues detectable by that experiment. The major inputs to model specification come from what we know of the anatomy, physiology and biochemistry of the system.

But given a model of a compartmental system, the first type of compartmental model, of a known size the structure may not be fully specified. The connectivities may not all be known. In that case we may have a set of possible models to examine for identifiability and parameter estimation. For the experiments that are run we have to generate a set of st ructurally equivalent models [1].

\section{IDENT IF IAB ILITY}

\subsection{Background}

For systems described by a vector of parameters the term identifiability refers to whether the information theoretically available from an experiment or set of experiments is sufficient to give a unique solution for the parameters. Unfortunately there is considerable variability in usage for a number of terms. Partly this arises from the multiple origins of the identification problem. The problem has been recognized in statistics, econometrics, control theory, communication theory, systems theory and in biology. Recently Nguyen and Wood [2] have reviewed inear identifiabilty; references to early papers in a number of fields will be found there. To my mind there are two major sources of confusion. One is that the term identification or system identification has a different meaning from identifiability. The former refers to the overall problem of determining system structure and parameter values from input-out put data whereas identifiability as usually used refers to the more narrow problem of the theoretical existence of unique solutions. But they are sometimes confused. The other is that the boundaries of the identifiability problem differ in different fields; some include observation error, some do not.
For applications in biology a significant step was the recognition that once the model was specified, estimation of parameters could be broken into at least two stages. In the first stage we ask, given the model and the compartments which can be injected and those which can be observed, are the parameters uniquely defined if we have error free observations? This is the identifiability problem for compartmental systems. It is useful to separate the effect of observation error from the purely mathematical question of existence of unique solutions. Obviously one would want to know whether the parameters were in theory uniquely defined by the observations before going into the parameter estimation phase. This division of the problem is particularly important for compartmental modeling in biology because we often do know the structure of the system and because the nature of biological systems limits the allowed experiments.

What of the effect of a finite number of discrete observations and of the truncatin of sampling time. It is common to put these into the next stage, parameter estimation, because of the interaction of these constraints with observation error so they must be included in questions of the design of experiments. However, one could argue for their inclusion in the deterministic identifiability problem or for splitting identifiability into a two stage question which takes them into account in the second stage.

The recognition of the utility of splitting the overall problem into stages came slowly. Kalman's work [3] showing that the impulse response matrix of a linear time invariant system identifies only the completely controllable and completely observable part of a dynamic system has played an important role, as have a number of others, see references in [2] and $[4,5]$. An early and important paper in compartmental analysis that took up problems of parameter estimability and foreshadowed some of the modern ideas on identifiability was that of Berman and Schoenfeld [6]. But the first statement of the identifiability problem in the compartmental context comes from Bellman and Astrom [7] who introduced the term structural identifiability.

The deterministic identifiability problem is so clean and has so many interesting facets it has drawn most of the attention in the last decade. So much so that at times it appears we have forgotten it is only one stage of the inverse problem. Bekey and Hadaegh [8] inveigh against deterministic identifiablity because of its neglect of modeling error and of observation error. However, it would seem preferable to keep deterministic

identifiability as a separate stage because of the insights it gives into problems and look at the effect of error as a separate issue. 


\subsection{Definitions and Terminology}

For the most part I shall use the terminology proposed by Cobelli and Distefano [9] who have reviewed the field with an eye to clearing up ambiguities of usage in applications to biological systems. A parameter is unidentifiable on an interval if there exist an infinite number of solution values and the system is unidentifiable if at least one of its parameters is unidentifiable. A parameter is identifiable on an interval if there are only a finite number of solution values in that interval and it is uniquely identifiable if there exists only one solution. A system is identifiable if all of its parameters are either identifiable or uniquely identifiable and at least one of the parameters is only identifiable. Finally, a system is uniquely identifiable if all parameters are uniquely ident ifiable.

Other important terms are local and global identifiability. Local identifiability refers to uniqueness of solution in a small neighborhood of a point in parameter space. If the solution is unique for the entire parameter space we have global identifiability.

If stochastic considerations are included one can define maximum likelihood (ML), least squares (LS) and consistency-in-probability (CP) identifiability [2]. For a class of linear time invariant stochastic or deterministic systems Nguyen and Wood [2] show that local ML, LS, and CP identifiability are equivalent to local parameter identifiability of the deterministic systems.

\subsection{System and Experiment}

It is important to keep clearly in mind the distinction between the system and the experiment wo do on the system. For compartmental systems, linear or nonlinear, but with coefficients independent of time, a very common type of experiment is to have the system in a steady state and to inject a tracer into one or more compartments and follow the time course of the tracer in one or more compartments. To take a simple example, the system diagram thay be $1(a)$ in which $i_{1}$ is a

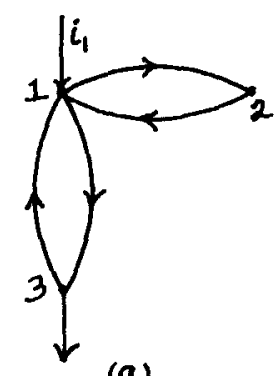

(a)

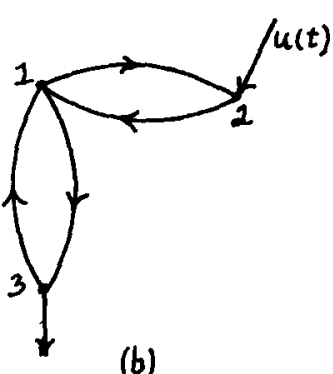

Figure 1 constant input into compartment 1 . If the experiment consists of an injection of tracer into 2 and observations of specific activity in 3 , the connectivity diagram for tracer flow will be $1(b)$. The equations for the system are (1) in which $q$ is the vector of amounts of

$$
\dot{q}=\mathrm{fq}_{\mathrm{q}}+\dot{\mathrm{i}}, \mathrm{q}(0)=\mathrm{q}_{\mathrm{o}}
$$

material in the compartments and $f$ is the matrix of fractional transfer coefficients. Let $x$ be the vector of tracer amounts in the compartments and let $y$ be the vector of observations. Then the equations for the tracer experiments are (2). It is useful to

$$
\begin{aligned}
& \dot{x}=f x+u, \quad x(0)=0 \\
& y=\left[\begin{array}{l}
0 \\
0 \\
y_{3}
\end{array}\right], y_{3}=x_{3} / q_{3}
\end{aligned}
$$

distinguish the experiment by using double arrows for experimental input and for observations and to superimpose these on the system connectivity diagram as in Figure 2 .

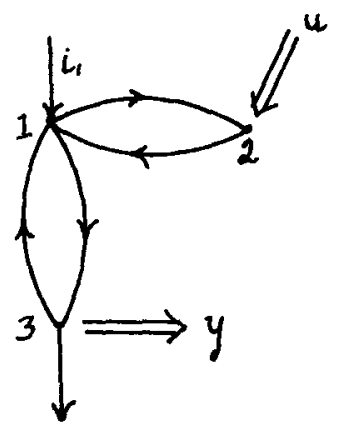

Figure 2

In general then the equations of an experiment will consist of the homogeneous equations for the system plus the inputs of the experiment, initial conditions, the equations for the observations and equations for any other constraints. The first three are given in equations (3). Here $u$ is an input vector for

$$
\begin{aligned}
& \dot{x}=f x+B u, \quad x(0)=x_{0} \\
& y=C x
\end{aligned}
$$

the experiment and $B$ is the matrix which defines the inputs to the different compartments, $C$ is the observation matrix. In general system theory it is common to write $y$ in the form $y=C x+D u$ but the last term so rarely enters the observations for compartmental systems there is no point to carrying it. 
In compartmental analysis usually all of the elements of $B$ are known and the zeros of $f$ are known because we know the digraph for the system. But not all of the elements of $\mathrm{C}$ are always known; unknown elements occur when we measure a specific activity or concentration of a tracer in a sample, i.e. $x_{j} / q_{j}$ or $x_{j} / V_{j}$, and we don't know the dilution by abundant isotope, $q_{j}$, or the volume of distribution, $v_{j}$. In addition there may be constraints that take into account prior information on the system.

\subsection{Necessary Conditions}

\subsubsection{Input and Output Reachability}

For this section assume the only unknown parameters are the non-zero elements of the system matrix, $f$. The most general sort of condition that must be satisfied is that the inputs must excite all of the modes of the system and all of the modes must appear in the observations. The first can be put in terms of input reachability; al1 compartments must have at least one path leading to them from some input. The second is the condition of out put reachability. For all compartments other than terminal compartments (simple traps) there must be a path going from the compartment to one of the observed compartments. These conditions are easily checked by examining the reachability matrix of the system digraph. The terms input and output connectability $[10,11]$ have been used as well as input and output reachability [12]; I prefer reachability because it conforms with standard usage in graph theory.

Input and out put reachability are necessary conditions for all systems, linear and nonlinear.

\subsubsection{A Stronger Necessary Condition.}

A general necessary condition for all systems is that the observations and prior information provide at least $p$ relations in the $p$ parameters which have to be estimated. For linear systems this means that the transfer function for the input-output experiment plus the prior information not in the transfer function must provide at least $p$ equations in the $p$ parameters. If there are $p$ relations there is no guarantee that the system is uniquely identifiable or even identifiable. But. if $p$ relations cannot be obtained the system is unidentifiable. Cobel1i et al. [10] have published a condition for time invariant linear systems which was obtained by comparing the terms of the transfer function with properties of the system connectivity diagram to give a count of the number of non-redundant relations obtainable from the transfer function.

\subsection{Sufficiency}

Basically the identifiability problem for time invariant linear systems reduces to a question of the existence of a unique solution to a system of nonlinear equations. So far as $I$ know a general solution for this problem is not available. However a number of constructive approaches have been proposed which amount to recipes telling one how to determine whether or not a compartmental system is identifiable. All work well with small systems but the effort needed mounts rapidly with the size of the system.

Four major methods have been proposed; they are obviously related through the basic theory of linear compartmental systems.

\subsubsection{The Transfer Function or Impulse Response Method}

Bellman and Astrom [7] pointed out that if a system is identifiable from any input it is identifiable from the impulse response so Bellman and Astrom suggested that we examine the transfer function for the experiment. The method has been used by many and in particular by Cobe11i et a $1 .[10,13]$.

In theory the method is simple. Consider the system with experiment given by equations (3) but let us assume a zero initial state problem, i.e. $x_{0}=0$. Laplace transforms give us the set $(4)$.

$$
\begin{aligned}
& (s I-f) X=B U \\
& Y=C X \\
& Y=C(s I-f)^{-1} B U
\end{aligned}
$$

We $\operatorname{call} \mathrm{H}(\mathrm{s})=(\mathrm{sI}-\mathrm{f})^{-1}$ the transfer function for the system, $H_{i j}(s)$ gives the observation in compartment $i$ for unit impulse into compartment $j, c(s I-f)^{-1} B$ is the transfer function for the given experiment; it tells us what components or combinations of the elements of $\mathrm{H}(\mathrm{s})$ are available from the given experiment. The problem then reduces to looking at the coefficients of the terms of this transfer function to see if all of the components of $f$ are uniquely determined and this is a problem in simultaneous nonlinear equations.

Related to this is Eisenfeld's [14] use of integral transforms to generate irreducible representations. Eisenfeld includes the restriction that data are truncated in time so that instead of using Laplace transforms he goes to a finite sequence of general integral transforms over an interval. This restricts the identifiability problem by introducing one of the practical constraints on data gathering.

\subsubsection{Markov Parameters}

The impulse response in the experiment is given by equation (5). 


$$
y=C e^{f t} t_{B}
$$

Expanding $e^{f t}$ in a matrix infinite series gives equation (6).

$\mathrm{y}=\mathrm{CB}+\mathrm{CfBt}+\mathrm{Cf}^{2} \frac{\mathrm{B}^{\mathrm{t}^{2}}}{2 !}+\ldots+\mathrm{Cf}^{\mathrm{k}} \frac{\mathrm{Bt}^{\mathrm{k}}}{\mathrm{k} !}+\ldots$

The coefficients $C B, C f B, \ldots$ are the Markov parameters and we consider them to be obtainable from the impulse response. If $f$ is $\mathrm{N} \times \mathrm{N}$, we need consider only the first $\mathrm{N}$ of them; a recursion relation of degree $N+1$ defines the remainder [14]. Identifiability now amounts to asking whether the parameters are all defined by the Markov coefficients.

The relation to the transfer function method is then obvious if we write out the transfer function. The transfer function for the experiment is given by (7). $\Delta(s)$ is the determinant of $(S I-f)$.

$$
\begin{aligned}
& G(s)=C(s I-f)^{-1} B=\frac{C \operatorname{adj}(s I-f) B}{\Delta(s)} \\
& \Delta(s)=\operatorname{det}(s I-f)=s^{N}+\alpha_{1} s^{N-1}+\ldots \\
& +\alpha_{N-1} s+\alpha_{N}
\end{aligned}
$$

But $c \operatorname{adj}(s I-f) B$ can be written (15) in the form of eqn. (9).

$$
\begin{aligned}
C \operatorname{adj}(s I-f) B & =C B\left[s^{N-1}+\alpha_{1} s^{N-2}+\ldots+\alpha_{N-1}\right] \\
& +C f B\left[s^{N-2}+\alpha_{1} s^{N-3}+\ldots+\alpha_{N-2}\right] \\
& \cdot \\
& \cdot \\
& \cdot \\
& +C f^{N-2} B\left[s+\alpha_{1}\right] \\
& +C f^{N-1} B
\end{aligned}
$$

\subsubsection{Similarity Transformations and the Set} of Equivalent Models

An approach which I believe gives more insight into the problem is to seek all models which give the same input-output response for the given experiment and which satisfy the known constraints such as having the same connectivity diagram. It has been called exhaustive modeling by Walter and Le Courtier [16] and the direct method by Travis and Haddock [17]. The method was anticipated but not fully developed in the early work of Berman and Schoenfeld [6].

Given the system and experiment of equations (3) all systems equivalent to it must have a system matrix which is related to $f$ by a similarity transformation and which satisfies the constraints on the system matrix. Thus we seek a nonsingular matrix $P$ such that the equations for the experiment are (10).

$$
\begin{aligned}
& \dot{x}=\left(P^{-} 1_{f P}\right) x+B u \\
& y=C x
\end{aligned}
$$

Taking transforms we obtain (11) for $Y$.

$$
\mathrm{Y}=\mathrm{CP}^{-1}(\mathrm{sI}-\mathrm{F})^{-1} \mathrm{PBU}
$$

For the input-out put kinetics to remain the same, eqns. (12) hold. Relations (12)

$$
\mathrm{CP}-1=\mathrm{C} \quad, \quad \mathrm{PB}=\mathrm{B}
$$

determine some of the elements of $P$. Then apply the known constraints on the system matrix to $P^{-1} f_{f P}$ i.e. the zero elements and any relations between the non-zero elements, to try to determine the remaining elements of $P$. If $P=I$ is the only solution the system is uniquely identifiable. If $P=I$ is a solution and a row (column) permutation of $I$ is also a solution, it means there is only one set of values for the elements of the system matrix but there is a symmetry in system structure such that the compartments corresponding to the permuted rows play interchangeable roles [17]. If there is a finite set of solutions for $P \neq I$ the system is identifiable, otherwise it is unidentifiable.

An important extension of this occurs when the connectivity diagram is not fully known so we are back in the model specification phase. We then have the problem of generating the set of what Vajda [1] calls the structurally equivalent models for the input-output experiment. This is then a problem of generating realizations of an input-out put experiment under constraints.

\subsubsection{The Modal Matrix Approach}

Another approach is to identify the elements of the matrix of eigenvectors, the modal matrix, and the eigenvalues. Delforge $[18,19]$ and Norton [20] have used this idea but with different approaches to obtaining solutions.

The basic idea is simple. Again we start with the impulse response, eqn. (5). Let $M$ be the modal matrix and $\Lambda$ be the diagonal matrix of eigenvalues so $f=M \Lambda M^{-1}$. Hence we can write the impulse response.

$$
y(t)=C e^{M \Lambda} M^{-1} t_{B}=C M e^{\Lambda t_{M}-1} B=E e^{\Lambda t_{D}}
$$

If the $f$ matrix is pxp we have.

$$
f_{i j}=m_{1}^{I} \Lambda n_{j},
$$

where $m_{i}^{T}$ is row $i$ of $M$ and $n_{j}$ is column

j of $M^{-1}=N$. Also,

$f_{o j}=-\sum m \Lambda_{n j}$, and

$\mathbf{m i n}_{\mathbf{i}}=\delta_{i j}$ 
Because the eigenvectors are determined only to within a multiplicative constant we can set one element of each column of $M$ arbitrarily. Note that equations $(14)-(16)$ are bilinear relations in elements of $M$ and $N$. To these we add (17).

$$
x(t)=e^{f t} x(0)+\int_{0}^{t} e^{f(t-\tau)} B u(\tau) d \tau
$$

So for zero initial conditions and impulsive input for example, into $j$.

$$
\begin{aligned}
& x(t)=M e^{\Lambda} t_{n_{j}} \\
& x_{i}(t)=m_{i}^{T} e^{\Lambda} n_{j}
\end{aligned}
$$

Using the constraints on $f$ and equations (16) and (18) we try to determine the elements of $M$ and $N$. Delforge counts the number of nonredundant equations to see if the set as a whole provides a solution. Norton tries to determine the rows of $M$ and columns of $M^{-1}$ in a stepwise manner, using the known constraints to try to obtain a sequence of linear problems. The point to the latter is that as soon as a row of $M$ is known all bilinear forms in that row and any of the columns of $M^{-1}$ become 1 inear equations.

A11 of the methods just reviewed work well with small systems but become increasingly more difficult to use as system size increases. In theory all could be programmed but might be serious $1 y$ limited by computability bounds. An approach worth investigating is to look for a decomposition into subsystems in a way that allows one to say something about identifiability of the whole system from properties of the subsystems. Eisenfeld (21) has reported on some initial steps in this direction.

\subsection{Nonlinear Systems}

Much less has been done with nonlinear systems or with linear systems with time varying coefficients. Grewal and Glover (5) look at identifiability of nonlinear systems by looking at the linearized system. This fits in with the practical approach of the physiologist who puts nonlinear compartmental systems into steady states in which the movement of tracers follows linear kinetics. Pohjanpalo (22) however looks at identifiability of the derivatives at $t=0$ for a Maclaurin series expansion for nonlinear systems.

\section{PARAMETER EST IMAT ION}

\subsection{Identifiability, Conditional Estimation, Estimability}

It is important to look at identifiability first because of the information it gives about what an experiment can potentially tell us about a system. Nonetheless it is possible for a system to be unidentifiable and for its parameters to be restricted to small enough ranges for the system to be "identifiable for practical purposes". More importantly if a system is unidentifiable it is always possible to set a subset of the parameters to make it identifiable; estimates of the remaining parameters are then conditional estimates. For small parameter sets one can often obtain considerable insight into a system by plotting both the optimum values of the object function, the minimum of a sum of squares for example, and the values of the conditional estimates of the estmated parameters as functions of the values of the one or two parameters that are set arbitrarily.

It is also possible for a system to be identifioable for a given experiment but for the measurements to be so insensitive to variation in one or more of the parameters that their estimates have high variances and so they are poorly estimable. This impels us to consider a theory of estimability in which we take into account the restrictions on sampling, the errors of observations and possibly other contraints. The path to estimability is by way of studies of sensitivity and then to optimization of estimability, i.e. design of experiments.

\subsection{Sensitivity}

Given a function differentiable in the parameters the local or point sensitivities are the derivatives with respect to the parameters. In a global sense we are interested in the sensitivity of the observations, $y$, to change in the parameter values. As a first step this could be examined without taking into account errors of observation by looking at the functional dependence of the observations on the parameters, the fractional transfer coefficients, or by direct compution of solutions of the forward problem for sets of values of the parameters. But that can be a tall order; it means looking at sensitivities of the observations as functions of the parameters over the parameter space. But the sensitivities at the parameter estimates are the most important.

of more immediate importance are the variances of the parameter estimates and these are directly related to the sensitivity of the object function used to estimate the parameters; the sensitivities of the observations at the parameter estimates appear within the sensitivities of the object function. Let us use least squares estimators for illustration. For simplicity assume we have a one component observation vector $y(t, \theta)$, parameter vector $\theta$ and samples at $t_{j}, j=$ $1, \ldots, m$. The sum of squares of deviations is given by equation (19), in which $w_{j}$ is a

$$
s=\sum_{j} w_{j}\left[y_{j}-y\left(t_{j}, \theta\right)\right]^{2}
$$


weight at the $\mathrm{jth}$ sample point. Let $\theta_{0}$ be the value of the parameter vector at the minimum of the sum of squares. Expanding around the minimum gives us equation (20).

$\Delta S=S\left(\theta_{o}+h\right)-S\left(\theta_{o}\right)=h^{T} G_{o}+\frac{1}{2} h^{T} H_{o} h+o\left(h^{3}\right)$

$G_{O}$ is the gradient vector at $\theta_{0}$ and $H_{0}$ is the matrix of second derivatives, the Hessian, evaluated at $\theta_{0}$. By assumption $\theta_{0}$ is a minimum of $S$ so $G_{O}=0$ and $H_{O}$ is positive definite. For small $h, \Delta S$ is a quadratic function of $h$ which for fixed $\Delta S$ is an ellipsoid in parameter space with principal axes in general not along the components of $\theta$. To see how $\Delta S$ changes with $\theta$ we need to examine the eigenvalues of $\mathrm{H}_{0}$; the sum of squares is most sensitive to change in $\theta$ along the principal axis corresponding to the largest eigenvalue of $\mathrm{H}_{\mathrm{O}}$ and is least sensitive to change in $\theta$ along the principal axis corresponding to the smallest eigenvalue of $\mathrm{H}_{\mathrm{O}}$.

For maximum likelihood estimators a similar set of considerations leads to a matrix which is closely related to the Hessian of the LS formulation and whose expectation is the Fisher information matrix.

\subsection{Design of Experiments}

In general then we should like to maximize the sensitivity of the minimum of the sum of squares, to variation in the parameters. But given the experiment, the number of samples and the sample times the minimum of the sum of squares is set for any particular sample. In theory if we knew the probability densities of the observational errors, the expected values of the minimum sum of squares and of the Hessian are determined. What we want to do then is to vary the number of samples and the sample times, within the constraints on the experiment, so as to maximize the sensitivity of the minimum sum of squares. That optimization problem is what is meant when we talk of design of experiments.

In the context of compartmental systems let us talk about "degign of experiments" in terms of a given experiment, i.e. we have a

compartmental system of known structure and identifiable by a specific experiment. The problem statement is then as follows.

\section{Given:}

a) The parameter values (in reality, an est imate)

b) Sampling is restricted to a finite interval in time, usually $0<\mathrm{t}<\mathrm{T}$.

c) The number of samples is no more than some number, $\mathrm{N}$.

d) The variances of the sampling errors or their distributions are known.
Find the partition of sampling times that optimizes the expected value of some function of the variances of the parameter estimates that is our measure of sensitivity. This is obvious1y an iterative problem because we need to know the values of the parameters which are to be estimated. Let us put aside questions of costs or utilities, for the moment. One approach is to minimize the volume of the constant error ellipsoid around the parameter estimate. If $\gamma_{1}, \gamma_{2}, \ldots, \gamma_{p}$ are the eigenvalues of the Hessian, the volume of the ellipsoid of constant error around the minimum sum of squares is proportional to the product (21).

$$
P=\prod_{j=1}^{P}\left(\frac{1}{\gamma_{j}}\right)^{2}
$$

But because we would like all parameters, $\theta_{i}$, to be estimable with $\hat{\sigma}_{\theta_{i}} / \hat{\theta}_{i}$ within some given ranges we really want to minimize the volume of the ellipsoid but also constrain the allowable deviation from a sphere. The latter represents a constraint on the relative values of the maximum and minimum eigenvalues of the Hessian evaluated at the parameter values. These two criteria work in opposite directions and we do not have extensive experience from which to evaluate the trade-offs between them.

At a broader level, if we may do more than one experiment the optimization has to be extended over all possible experiments or combinations of experiments. If the system is not fully specified the problem is really on a new level. If all possible system configurations are identifiable by the experiment (s) then the optimizaton has to be done from a Bayesian viewpoint, taking into account prior probabilities of different possible system configurations.

\section{CONCLUSION}

There are still many interesting things to do in identifiability for compartmental systems. But from the perspective of the overall inverse problem we are far enough along so that the field would profit from an increase in effort on the design of experiments.

\section{REFERENCES}

[1] Vajda, s., Structural equivalence of linear systems and compartmental models, Math. Biosci. 55 (1981) 39-64.

[2] Nguyen, V.V. and Wood, E.F., Review and unification of linear identifiability concepts, SIAM Rev. 24 (1982) 34-51.

[3] Kalman, R.E., Mathematical description of linear dynamical systems, SIAM J. Control 1 (1963) 152-192.

[4] Glover, K. and Willems, J.C., Parameterizations of linear dynamical systems: Canonical forms and 
identifiability, IEEE Trans. Automatic Control 19 (1974) 640-646.

[5] Grewa1, M.S, and Glover, K., Ident ifiability of 1 inear and nonlinear dynamical systems, IEEE Trans. Autom. Control 21 (1976) 833-837.

[6] Berman, M. and Schoenfeld, R., Invariants in experimental data on linear kinetics and the formulation of models, J. Applied Phys. 27 (1956) 1361-1370.

[7] Bellman, R. and Astrom, K.J., On structural identifiability, Math. Biosci. 7 (1970) 329-339.

[8] Bekey, G.A. and Hadaegh, F.Y., Is deterministic identifiability a fraud?, Proc. X IMACS Congress on Systems Simul. and Scientif. Comput. (1982).

[9] Cobel1i, C. and Distefano III, J.J., Parameter and structural identifiability concepts and ambiguities: a critical review and analysis, Am. J. Physio1. 239 (1980) R7-R24.

$\{10\rceil$ Cobelli, C., Lepschy, A. and Roman in Jacur, G., Structural identifiabilitty of linear compartmental models, in $\mathrm{Hal}$ fon, $E$. (ed.), Theoretical Systems Ecology (Acad. Press, NY, 1979).

[11] Davison, E.J., Connectability and structural controllability of composite systems, Automatica 13 (1977) 109-123.

[12] Siljak, D.D., On reachability of dynamic systems, Int. J. Systems Sci. 8 (1977) 321-338.

[13] Cobelli, C., Lepschy, A. and Romanin Jacur, G., Identifiability results on some constrained compartmental systems, Math. Biosci. 47 (1979) 173-195.

[14] Eisenfeld, J., On identifiability of impulse response in compartmental systems, Math Biosci. 47 (1979) 15-34.

[15] Chen, C.T., Int roduction to Linear System Theory, (Holt, Rinehart and Winston, Inc., NY, 1970).

$[16]$ Walter, $E$. and Lecourtier,Y., Unidentifiable compartmental models:- What to do?, Math. Biosci. 56 (1981) 1-25.

[17] Travis, C.C. and Haddock, G., On structural identification, Math. Biosci. 56 (1981) 157-173.

[18] Delforge, J., New results on the problem of identifiability of a linear system, Math. Biosci. 52 (1980) 73-96.

[19] Delforge, J., Necessary and sufficient structural condition for local identifiability of a system with linear compartments, Math. Biosci. 54 (1981) 159-180.

[20] Norton, J.P., Normal-mode identifiability analysis of linear compartmental systems in linear stages, Math. Biosci. 50 (1980) 95-115.

[21] Eisenfeld, J., Structural identifiability criteria for large linear and nonlinear compartmental systems, Proc. X IMACS Congress on Systems simul. and scientif. Comput. (1982).
[22] Pohjanpalo, H., System identifiability based on the power series expansion of the solution, Math. Biosci. 41 (1978) 21-33. 